Draft version April 7, 2022

Preprint typeset using $\mathrm{IAT}_{\mathrm{E}} \mathrm{X}$ style emulateapj v. 05/04/06

\title{
THE CORE COMPOSITION OF A WHITE DWARF IN A CLOSE DOUBLE DEGENERATE SYSTEM *
}

\author{
S. VEnNES ${ }^{1,2}$, A. KAWKA ${ }^{1,2}$ \\ ${ }^{1}$ Astronomický ústav, Akademie věd České republiky, Fričova 298, CZ-251 65 Ondřejov, Czech Republic \\ Draft version April 7, 2022
}

\begin{abstract}
We report the identification of the double degenerate system NLTT 16249 that comprises a normal, hydrogen-rich (DA) white dwarf and a peculiar, carbon-polluted white dwarf (DQ) showing photospheric traces of nitrogen. We disentangled the observed spectra and constrained the properties of both stellar components. In the evolutionary scenario commonly applied to the sequence of DQ white dwarfs, both carbon and nitrogen would be dredged up from the core. The $\mathrm{C} / \mathrm{N}$ abundance ratio $(\approx 50)$ in the atmosphere of this unique DQ white dwarf suggests the presence of unprocessed material $\left({ }^{14} \mathrm{~N}\right)$ in the core or in the envelope. Helium burning in the DQ progenitor may have terminated early on the red-giant branch after a mass-ejection event leaving unprocessed material in the core although current mass estimates do not favor the presence of a low-mass helium core. Alternatively, some nitrogen in the envelope may have survived an abridged helium-core burning phase prior to climbing the asymptotic giant-branch. Based on available data, we estimate a relatively short orbital period $(P \lesssim 13 \mathrm{hrs})$ and on-going spectroscopic observations will help determine precise orbital parameters.
\end{abstract}

Subject headings: binaries: close — stars: individual (NLTT 16249) — white dwarfs

\section{INTRODUCTION}

The presence of a large concentration of carbon, detected via $\mathrm{C}_{2}$ molecular bands, in the otherwise heliumrich atmosphere of many old $\left(\gtrsim 10^{9}\right.$ years) white dwarfs has been viewed for many years (e.g., Vauclair \& Fontaine 1979; Koester et al. 1982) as evidence of the carbon-rich nature of their cores: The material diffuses upward, away from the core, and is dredged-up to the surface by the deep helium convection zone developing in cool white dwarfs (Fontaine et al. 1984; Pelletier et al. 1986; MacDonald et al. 1998). These objects, collectively known as DQ white dwarfs, form a homogeneous sequence of stars with surface temperatures ranging from $\sim 11,000$ to $\sim 5,000 \mathrm{~K}$ and with temperature-correlated surface carbon abundances between $10^{-2}$ and $10^{-7}$ relative to helium by number (Dufour et al. 2005; Koester \& Knist 2006).

Evolutionary models (e.g., Schaller et al. 1992) show that stars of low to intermediate masses, i.e., below 8 to $10 M_{\odot}$, end their nuclear burning history with a core composed primarily of carbon and oxygen or heavier elements ( $\mathrm{Ne}, \mathrm{Mg}$ ) in the upper mass range (GarciaBerro et al. 1997). In the past, spectroscopic searches for oxygen (ultraviolet $\mathrm{CO}$ bands) or nitrogen (violet $\mathrm{CN}$ bands) have yielded abundance limits $\mathrm{O} / \mathrm{C} \approx 0.1-1$ increasing with temperature (Koester et al. 1982) or $\mathrm{O} / \mathrm{C}, \mathrm{N} / \mathrm{C} \approx 10^{-2}-10^{-3}$ (Wegner \& Yackovich 1984). Although oxygen is a primary product of the triple-alpha (3- $\alpha)$ nuclear burning process, the actual amount of oxygen dredged-up along with carbon from the interior depends on its abundance profile in the core (MacDonald et

* Based on observations collected at the European Organisation for Astronomical Research in the Southern Hemisphere, Chile under programme ID 086.D-0562.

${ }^{2}$ Visiting Astronomer, Kitt Peak National Observatory, National Optical Astronomy Observatory, which is operated by the Association of Universities for Research in Astronomy (AURA) under cooperative agreement with the National Science Foundation. al. 1998). On the other hand, nitrogen should be fully exhausted along with helium by the 3 - $\alpha$ process and is not expected in the atmosphere of white dwarfs with a fullyevolved core. Recently, Gänsicke et al. (2010) reported the discovery of two oxygen-rich white dwarfs alongside the carbon-rich DQ sequence. The measured $\mathrm{O} / \mathrm{C}$ abundance ratios $(\log \mathrm{O} / \mathrm{C} \approx 0.6-1.8)$ imply core compositions dominated by oxygen ( 80 to $99 \%$ by mass). The stars were suspected by Gänsicke et al. (2010) to be the descendents of the most massive stars to avoid core collapse $\left(9-10 M_{\odot}\right.$; Garcia-Berro et al. 1997).

Clearly, the surface composition reflects the white dwarf core composition which, in turn, is determined by the evolutionary path followed by the progenitor. In this context, we present in Section 2 spectroscopic observations of the peculiar object NLTT 16249 that show a strong Balmer line series along with $\mathrm{C}_{2}$ Swan and $\mathrm{CN}$ violet band absorptions. The observed radial velocity variations and a successful spectral decomposition and model atmosphere analysis (Sections 3.1-3.3) show that the object is in fact a close double degenerate system comprising a normal DA white dwarf and a peculiar DQ of similar optical luminosities. We examine possible evolutionary scenarios in Section 3.4, and we propose that the photospheric carbon to nitrogen abundance ratio $(\mathrm{C} / \mathrm{N} \approx 50)$ indicates that the core material was left partially unprocessed or that burning ceased before destroying all nitrogen in the envelope. Finally, we summarize in Section 4.

\section{OBSERVATIONS AND CLASSIFICATION}

We first observed NLTT 16249 on UT 2010 Mar 26 with the R-C spectrograph attached to the $4 \mathrm{~m}$ telescope at Kitt Peak National Observatory. We obtained three consecutive exposures of $900 \mathrm{~s}$ each using the KPC-10A grating with the WG360 order blocking filter. The spectra cover a wavelength range from 3660 to $6790 \AA$ with a dispersion of $2.8 \AA$ per pixel. This low dispersion spec- 


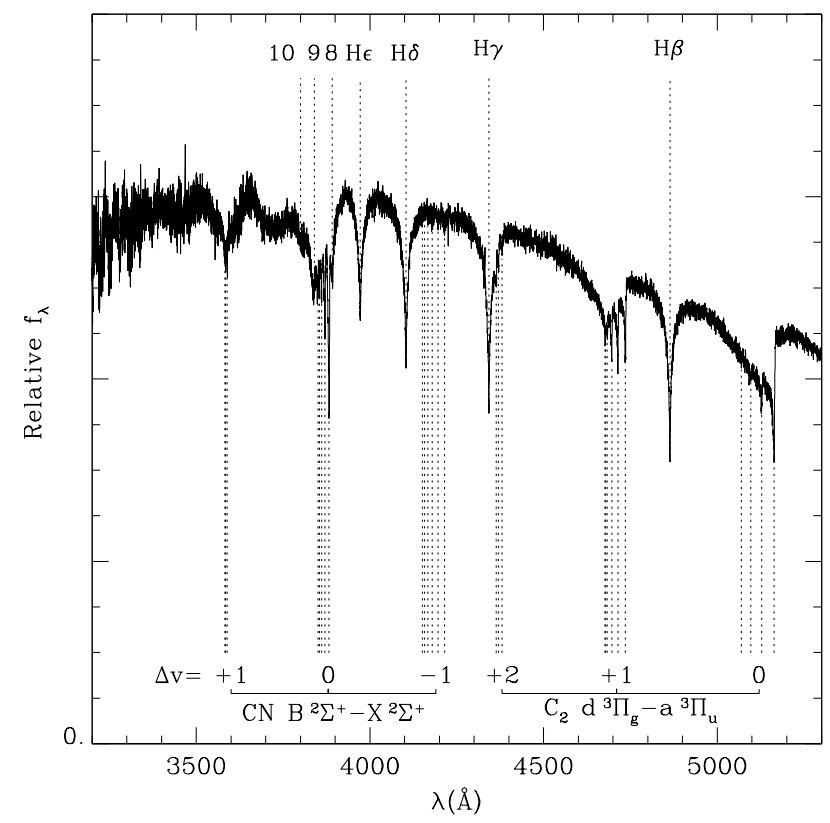

FIG. 1.- Spectrum of NLTT 16249 obtained with the UVB arm of X-shooter. The main spectral features (Balmer lines and $\mathrm{C}_{2}$ or $\mathrm{CN}$ molecular bands) are marked with dashed lines and labelled accordingly (see text)

trum $(\lambda / \Delta \lambda \approx 1000)$ revealed a spectroscopic combination that would be unusual in any star. The hydrogen Balmer line series are blended with $\mathrm{C}_{2}$ Swan bands (the $d^{3} \Pi_{g}-a^{3} \Pi_{u}$ system; see Tanabashi et al. 2007) and what appears to be the $\Delta v=0$ sequence of the $\mathrm{CN}$ violet band near $3880 \AA$ (the $B^{2} \Sigma^{+}-X^{2} \Sigma^{+}$system; see Ram et al. 2006).

Next, we obtained a single echelle spectrum on UT 2010 Nov 7 (2400 s exposure) with the X-shooter spectrograph (Vernet et al. 2011) attached to the UT2 (Kueyen) at Paranal Observatory. The spectrum covers a wavelength range from $3000 \AA$ to $2.5 \mu \mathrm{m}$ on three separate arms with a resolving power $\lambda / \Delta \lambda \approx 9100$ for the UVB arm, 8800 for the VIS, and 6200 for the NIR. This intermediate dispersion spectrum confirmed our earlier findings and also revealed the presence of additional sequences from the $B-X \mathrm{CN}$ system.

The star could be identified as a DQAZ, i.e., a heliumrich white dwarf with traces of carbon and hydrogen along with additional elements such as nitrogen, although the strength of the hydrogen lines is incompatible with this interpretation. More likely, NLTT 16249 is a $\mathrm{DA}+\mathrm{DQ}(\mathrm{N})$ double degenerate system comprised of a normal hydrogen-rich (NLTT 16249B) and a nitrogencontaminated DQ white dwarf (NLTT 16249A). We propose a non-standard classification for the DQ $(\mathrm{N})$ white dwarf owing to the unforeseen presence of nitrogen (for the current classification scheme see Sion et al. 1983). The diagnostics employed to confirm the second possibility are, first, a radial velocity study and, next, a spectral decomposition into two components.

Figure 1 shows the X-shooter spectrum of NLTT 16249 (UVB arm): The main molecular bands are marked at selected wavelengths (Wallace 1962) following standard spectroscopic notations along with the Balmer lines.

The relative velocity change between the two compo-
TABLE 1

Adopted $\mathrm{C}_{2}$ AND CN BANDhead and $g f$ values

\begin{tabular}{ccccccl}
\hline \hline & & \multicolumn{2}{c}{$\mathrm{C}_{2}$} & & \multicolumn{2}{c}{$\mathrm{CN}$} \\
\cline { 7 - 7 } \cline { 6 - 7 }$\Delta v$ & $\left(v^{\prime}, v^{\prime \prime}\right)$ & $\lambda^{\mathrm{a}}(\AA)$ & $g f^{\mathrm{b}}$ & & $\lambda^{\mathrm{a}}(\AA)$ & $g f^{\mathrm{c}}$ \\
\hline-1 & $(0,1)$ & 5635.5 & 0.0198 & & 4216.0 & 0.00497 \\
& $(1,2)$ & 5585.5 & 0.0231 & & 4197.2 & 0.00826 \\
& $(2,3)$ & 5540.7 & 0.0192 & & 4181.0 & 0.0103 \\
& & & & & \\
0 & $(0,0)$ & 5165.2 & 0.0783 & & 3883.4 & 0.0684 \\
& $(1,1)$ & 5129.3 & 0.0300 & & 3871.4 & 0.0560 \\
& $(2,2)$ & 5097.7 & 0.0123 & & 3861.9 & 0.0466 \\
& & & & & \\
+1 & $(1,0)$ & 4737.1 & 0.0300 & & 3590.4 & 0.00767 \\
& $(2,1)$ & 4715.2 & 0.0435 & & 3585.9 & 0.0132 \\
& $(3,2)$ & 4697.6 & 0.0435 & & 3583.9 & 0.0170 \\
& & & & & \\
+2 & $(2,0)$ & 4382.5 & 0.0045 & $\ldots$ & $\ldots$ \\
& $(3,1)$ & 4371.4 & 0.0102 & $\ldots$ & $\ldots$ \\
& $(4,2)$ & 4365.2 & 0.0130 & $\ldots$ & $\ldots$ \\
\hline
\end{tabular}

a From Wallace (1962).

${ }^{\mathrm{b}}$ From Zeidler-K.T. (1987).

${ }^{\mathrm{c}}$ From Bauschlicher et al. (1988).

nents was readily measurable by using spectra obtained at the two available epoches, although individual velocity changes may suffer from large uncertainties on the zero-point of the low-dispersion KPNO wavelength scale. The relative velocity change between the two components is free of systematic errors and allowed us to estimate $K_{\mathrm{DQ}}+K_{\mathrm{DA}} \gtrsim 320 \pm 30 \mathrm{~km} \mathrm{~s}^{-1}$. On the other hand, the velocity shift of the DQ component was $v_{1, \mathrm{DQ}}-v_{2, \mathrm{DQ}}+C=120 \pm 30 \mathrm{~km} \mathrm{~s}^{-1}$, where the subscripts "1" and "2" refer to the KPNO and VLT spectra, respectively, and $C$ is the zero-point error. Similarly, for the DA component $v_{1, \mathrm{DA}}-v_{2, \mathrm{DA}}+C=-200 \pm 30 \mathrm{~km} \mathrm{~s}^{-1}$, i.e., in opposite direction to the DQ component. The amplitude ratio would imply that the DA white dwarf is more massive than its companion with $M_{\mathrm{DQ}} / M_{\mathrm{DA}}=1.7_{-0.6}^{+0.9}$, but because of potential systematic errors we will simply assume that $M_{\mathrm{DQ}} \approx M_{\mathrm{DA}}$.

The binary has a significant proper motion of

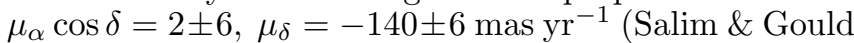
2003 ) in a relatively crowded field. We collected Johnson photometry $(V=15.77, B-V=0.24, U-B=-0.66$ with an assumed error of 0.02 on the colors) from Eggen (1968) and 2MASS photometry $(J=14.87 \pm 0.04)$ from Skrutskie et al. (2006). A close examination of the VLT acquisition image (epoch 2010) shows a fainter crowded star to the NW. Taking into account the proper motion of NLTT 16249, the stars would have nearly overlapped circa 1990. The $2 \mathrm{MASS}-J$ frame (epoch 1997) clearly shows a SE-NW elongation. Also, the DSS1 (epoch 1955) red and blue plates show that the crowded star is markedly red so that it probably contaminated the 2MASS- $J$ photometric measurement. With the crowded star at a larger separation, the UBV photometry obtained by Eggen (1968) circa 1965 was probably not significantly contaminated. In order to extend available broadband indices further in the red, we folded the X-shooter UVB and VIS spectra with VRI bandpasses (Bessell 1990) and measured $V-R=0.15$ and $V-I=0.37$.

\section{ANALYSIS}



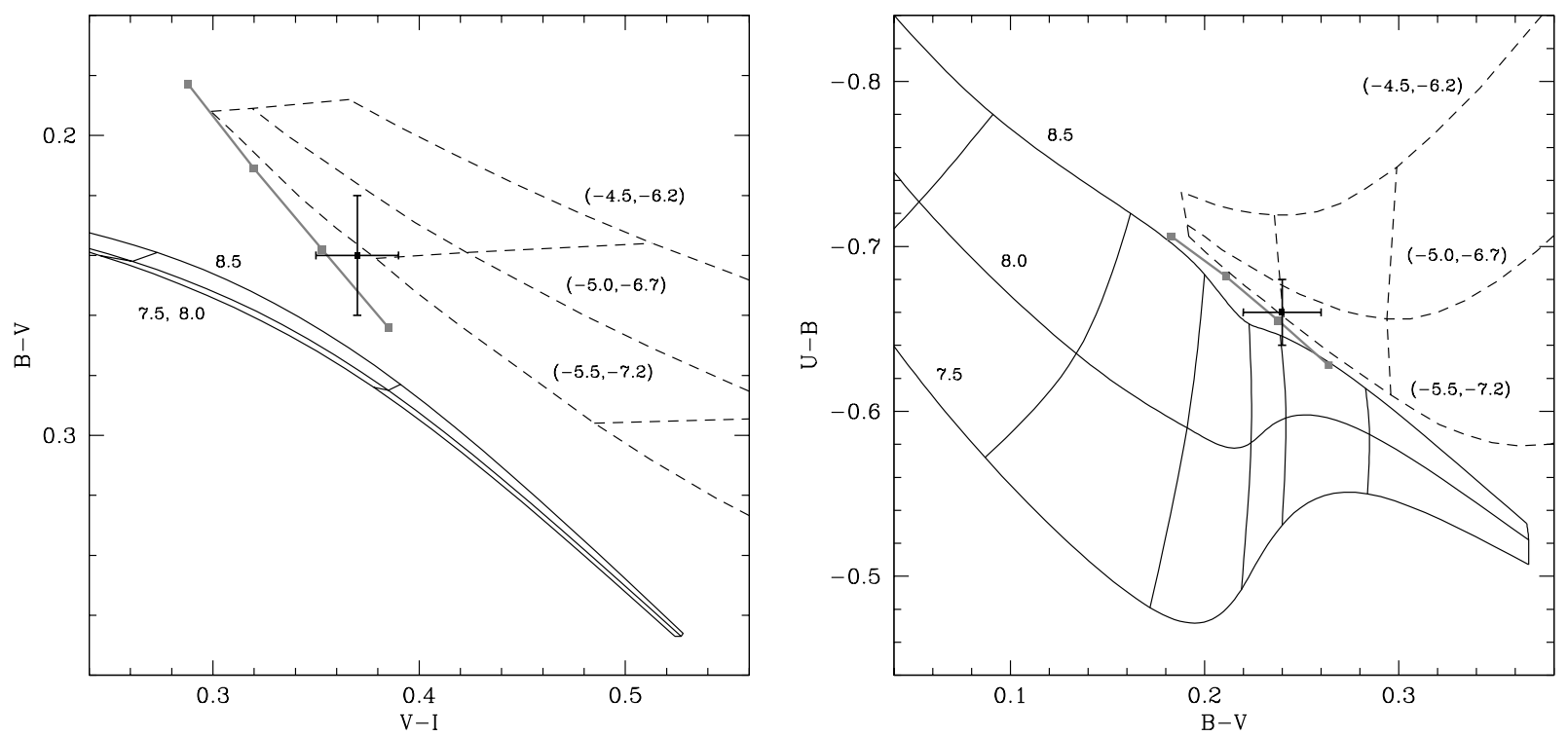

FIG. 2.- Johnson-Cousin color indices $B-V$ versus $V-I$ (left panel) and $U-B$ versus $B-V$ (right panel) for pure-hydrogen (DA) model grids (full lines) and DQ model grids (dashed lines). The DA model grids are shown with varying surface gravity (labelled at $\log g=7.5,8.0$, and 8.5) and temperature (shown from right to left at $7,8,9,10,12,14,16 \times 10^{3} \mathrm{~K}$ ). The DQ model grids are shown with varying carbon and nitrogen abundance (labelled with the pair $\log \mathrm{C} / \mathrm{He}, \log \mathrm{N} / \mathrm{He}$ ) and temperature (shown from right to left at 7 , $\left.7.5,8 \times 10^{3} \mathrm{~K}\right)$ at $\log g=8$. On both panels the observed colors are shown with error bars, and the best-fit model colors are shown, from upper-left to lower-right, with grey squares and lines at $(\log \mathrm{C} / \mathrm{He}, \log \mathrm{N} / \mathrm{He})=(-4,-5.7),(-4.5,-6.2),(-5.0,-6.7),(-5.5,-7.2)$.

The components were analyzed conjointly with the total flux measured at earth given by

$$
f_{\nu}=\frac{4 \pi}{D^{2}}\left(R_{\mathrm{DQ}}^{2} H_{\nu, \mathrm{DQ}}+R_{\mathrm{DA}}^{2} H_{\nu, \mathrm{DA}}\right)
$$

where $D$ is the distance to the binary, $R$ their respective radii, and $H_{\nu}$ their respective model Eddington fluxes. The individual radii, hence masses, are constrained by the surface gravity of each model grid point using massradius relations from Benvenuto \& Althaus (1999). The best-fit parameters $\left(T_{\text {eff,DQ }}, \log g_{\mathrm{DQ}}, T_{\text {eff,DA }}, \log g_{\mathrm{DA}}\right.$, and $\mathrm{DQ}$ abundances $\mathrm{C} / \mathrm{He}, \mathrm{N} / \mathrm{He}$ ) are obtained using $\chi^{2}$ minimization techniques. We also computed JohnsonCousin UBVRI magnitudes and color indices of the composite spectra to constrain the best-fit parameters. Because of the absence of $\mathrm{CO}$ spectroscopic signatures we set the oxygen abundance to zero in the DQ models. Moreover, the absence of $\mathrm{Ca} \mathrm{H} \& \mathrm{~K}$ and $\mathrm{CH}$ G-band also suggests very low metallicity in either star and the absence of hydrogen in the DQ white dwarf. With the revealing exception of nitrogen the $\mathrm{DQ}$ component appears normal.

\subsection{Model atmospheres}

The DA model atmospheres are described in Kawka \& Vennes (2006, 2011). The new DQ model atmospheres are in dual convective/radiative equilibrium as well as in local thermodynamic equilibrium. We included all relevant species $\left(\mathrm{He}, \mathrm{He}^{+}, \mathrm{C}, \mathrm{C}^{+}, \mathrm{N}, \mathrm{N}^{+}, \mathrm{O}, \mathrm{O}^{+}\right)$and molecules $\left(\mathrm{C}_{2}, \mathrm{CN}, \mathrm{CO}\right)$ in the charge conservation and abundance equations: This non-linear system of equations was solved using the secant method before each model iteration. We employed the molecular partition functions of Sauval \& Tatum (1984). We also estimated the contributions of $\mathrm{C}_{2}^{+}$and concluded that virtually all electrons are contributed by the ionization of helium and carbon.
The models add all relevant opacities, including ultraviolet $\mathrm{C}$ I lines, and the $\mathrm{C}_{2}$ Swan bands $(\Delta v=$ $-1,0,+1,+2)$ and the violet $\mathrm{CN}$ bands $(\Delta v=-1,0,+1)$ using the "just-overlapping line approximation" (Golden 1967; Zeidler-K.T. \& Koester 1982). We also added the $\mathrm{He}^{-}$free-free and the $\mathrm{C}^{-}$bound-free and free-free opacities as well as helium Rayleigh scattering. We updated the Zeidler-K.T. (1987) CN molecular opacities with oscillator strengths from Bauschlicher et al. (1988) and molecular constants tabulated in Reddy et al. (2003) in good agreement with Ram et al. (2006). Table 1 lists adopted bandhead wavelengths and $g f$ values for the first three vibrational transitions of the $\mathrm{C}_{2}$ and $\mathrm{CN}$ systems.

Figure 2 shows relevant photometric properties of the model grids. In particular, optical DQ colors are sensitive to carbon (and nitrogen) abundance because of varying depths of Swan and CN violet bands. This effect influences the composite colors and the selection of best-fit composite models. We found that the DQ spectral energy distribution is only mildly affected by surface gravity variations (see Dufour et al. 2005). The DQ synthetic colors are markedly bluer than those of a DA model at the same temperature.

\subsection{Properties of the components}

First, we proceed with the spectral decomposition. We mapped the minimum $\chi^{2}$ values in the $\left(\log g_{\mathrm{DQ}}, \log \mathrm{C} / \mathrm{He}\right)$ plane corresponding to the best-fit DA parameters $\left(T_{\text {eff,DA }}, \log g_{\mathrm{DA}}\right)$ and DQ temperature $\left(T_{\text {eff,DQ }}\right)$. We varied $\log \mathrm{C} / \mathrm{He}$ from -5.5 to -4.0 and $\log g_{\mathrm{DQ}}$ from 7.5 to 8.75 . In all spectral decompositions the nitrogen abundance scaled with the carbon abundance with a ratio $\log \mathrm{C} / \mathrm{N} \approx 1.7$ that we subsequently held fixed at this value. The calculations show that a family of solutions exists along an axis in the $\left(T_{\text {eff,DQ }}, \log \mathrm{C} / \mathrm{He}\right)$ plane where higher DQ temperatures 

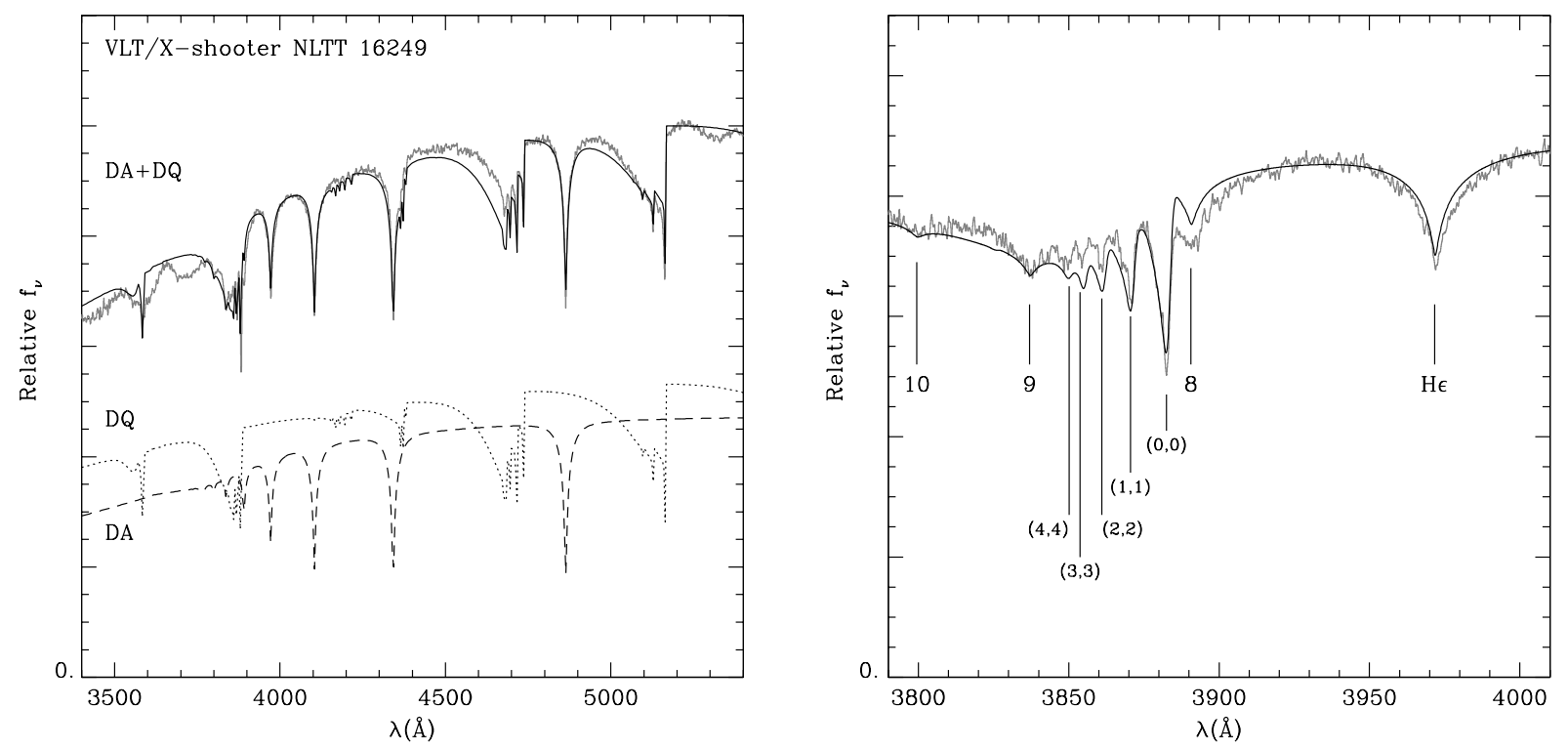

FIG. 3.- (Left panel) Model decomposition of the observed spectrum (degraded to a resolution of $2.4 \AA$ for clarity). The model flux contributions of the DA and DQ components are shown with dashed and dotted lines, respectively. The total DA+DQ model flux (full line) is compared to the observed flux (grey line). (Right panel) Composite model spectrum fit to the X-shooter spectrum (full resolution) near the Balmer series limit and the $\mathrm{CN}$ violet band $(\Delta v=0)$.

are compensated with higher carbon abundances. This degeneracy of the solutions may be lifted by comparing the predicted colors of the model composites with observed colors. On the other hand, the minimum $\chi^{2}$ values in the $\left(\log g_{\mathrm{DQ}}, \log \mathrm{C} / \mathrm{He}\right)$ plane are steadily found for $\log g_{\mathrm{DA}} \approx 8.2-8.5$. Interestingly, the best-fit surface gravities (hence radii) always indicated similar binary component luminosities, so that the DQ gravity correlates with the DA gravity and was found in the range $\log g_{\mathrm{DQ}} \approx 8.0-8.5$.

The predicted color indices were compared to observed indices to constrain possible solutions. Figure 2 shows composite colors corresponding to the best-fit models with $\log \mathrm{C} / \mathrm{He}$ varying from -5.5 to -4.0 . As noted above, higher carbon abundances are compensated with high DQ temperatures. Both sets of indices favor a lower carbon abundance

$$
\log \mathrm{C} / \mathrm{He}=-5.0 \pm 0.3, \log \mathrm{N} / \mathrm{He}=-6.7 \pm 0.3,
$$

and we conclude that the DA model parameters are

$$
T_{\text {eff }, \mathrm{DA}}=8330 \pm 200 \mathrm{~K}, \log g_{\mathrm{DA}}=8.37 \pm 0.15,
$$

and, correlating with the DA parameters, the DQ parameters are

$$
T_{\text {eff,DQ }}=7770 \pm 200 K, \log g_{\mathrm{DQ}}=8.27 \pm 0.22 .
$$

The total system mass varies between 1.33 , in the lower gravity range, to $1.83 M_{\odot}$, in the higher gravity range. The distance calculated using the $V-M_{V}$ modulus varies from $\sim 34 \mathrm{pc}$ for the high system mass to $\sim 46 \mathrm{pc}$ for the low system mass. A parallax measurement would help confirm and narrow down the range of spectroscopic solutions.

Assuming a system mass in the range $M_{\mathrm{DA}}+M_{\mathrm{DQ}}=$ $1.3-1.8 M_{\odot}$ and a minimum velocity amplitude $K_{\mathrm{DA}}+$ $K_{\mathrm{DQ}} \gtrsim 320 \mathrm{~km} \mathrm{~s}^{-1}$, the orbital period is constrained to

$$
P=2 \pi G \frac{M_{\mathrm{DA}}+M_{\mathrm{DQ}}}{\left(K_{\mathrm{DA}}+K_{\mathrm{DQ}}\right)^{3}} \sin ^{3} i \lesssim 9.2-12.8 \text { hrs. }
$$

Owing to the large velocity amplitude and relatively short period, the orbital parameters $\left(P, e, K_{\mathrm{DA}}, K_{\mathrm{DQ}}\right)$ should be easy to determine.

Figure 3 shows the best-fit composite model spectrum to the X-shooter spectrum using parameters listed above. The Swan $\Delta v=+1$ bands appear too strong relative to $\Delta v=0$ bands, particularly in the extended wing. Fortunately, the abundance offset between these two bands does not exceed $\approx 0.2$ dex.

\subsection{The dominant carbon isotope}

The isotopic shift of the $\Delta v=+1$ band of ${ }^{13} \mathrm{C}^{12} \mathrm{C}$ relative to that of $\mathrm{C}_{2}$ is $7.5 \AA$ (Wegner 1984). By attributing the existing band to $\mathrm{C}_{2}$ we conclude that the absence of red-shifted absorptions implies $\mathrm{C}_{2} /{ }^{13} \mathrm{C}^{12} \mathrm{C} \gtrsim 10$, i.e., ${ }^{12} \mathrm{C} /{ }^{13} \mathrm{C} \gtrsim 10$.

\subsection{Evolutionary scenarios}

Hydrogen-deficient white dwarfs (DB and DQ white dwarfs, see Sion et al. 1983) are possibly the product of a late thermal pulse. This process leads to the formation of a hydrogen-deficient surface that is also enriched in carbon and oxygen (Herwig et al. 1999, and references therein). After reaching the white dwarf cooling sequence these objects develop a nearly pure-helium surface and are recognized as DB white dwarfs. While cooling further they develop a deep helium-dominated convection zone dredging-up carbon from the core (Fontaine et al. 1984; Pelletier et al. 1986; MacDonald et al. 1998).

The material mixed in the helium-dominated convection zone of the DQ white dwarf NLTT 16249A is enriched with carbon and nitrogen. The diffusion timescales at the bottom of the helium convection zone of $\mathrm{a} \sim 8000 \mathrm{~K}$ white dwarf are relatively short $\left(\approx 10^{3}\right.$ yr; Koester 2009). Therefore, a steady-state abundance of nitrogen and carbon is achieved only if the carbon/nitrogen-rich material is supplied to the envelope from the core. This scenario, commonly applied to ordi- 
nary carbon-rich DQ white dwarfs, should also apply to the peculiar DQ NLTT 16249A, but the source of nitrogen remains elusive.

A copious amount of nitrogen is produced in intermediate-mass stars between pulses on the AGB when the convective envelope reaches the hydrogenburning shell (Lattanzio et al. 1996). This production is deemed responsible for nitrogen enrichment in planetary nebulae (Karakas et al. 2009), but what fraction of it would remain within the helium envelope and contribute to the white dwarf core composition is an open question.

Nitrogen leftover material is present in the early-stages of helium burning on the horizontal-branch (HB), but it is quickly destroyed along with helium leading to the formation of a carbon/oxygen-rich core. For example, a carbon-to-nitrogen ratio of $\approx 50$ is briefly achieved in the core of $2 M_{\odot}$ HB star after $\sim 110 \mathrm{Myr}$, but it rapidly declines afterward (Schaller et al. 1992). At this particular stage the core composition is $64.4 \% \mathrm{He}, 27.6 \% \mathrm{C}, 0.6 \% \mathrm{~N}$, and $7.4 \% \mathrm{O}$ by mass, but by the end of the helium burning phase the remainder consists mostly of oxygen $(83.6 \%$ by mass) and carbon (12.7\%) without a trace of nitrogen. Prior to helium ignition and on the RGB the carbonto-nitrogen number ratio in the core is only $\approx 1 / 100$. At higher masses and up to $9 M_{\odot}$ nitrogen is destroyed even more rapidly during the core helium-burning phase, and on the RGB the carbon-to-nitrogen number ratio in the core never rises above $\approx 1 / 40$. Should burning be terminated while on the RGB, nitrogen would dominate carbon in a helium-rich core.

We found that the DQ NLTT 16249A is part of a close double degenerate system. Binary evolutionary scenarios (e.g., Nelemans et al. 2001) show that, normally, the mass of helium-core white dwarfs correlate with the double-degenerate orbital period and almost never exceed $0.4 M_{\odot}$. Our spectral decomposition suggests a DQ mass larger than $\approx 0.6 M_{\odot}$. Applying the SchönbergChandrasekhar mass limit to the case of a helium core and helium/hydrogen envelope, the core mass on the RBG could reach $M_{c} / M=0.08$ prior to collapse and ignition, i.e., $M_{c} \approx 0.6 M_{\odot}$ in the case of a $M=8 M_{\odot}$ star. In order to conceal a large helium-rich core the DQ white dwarf in NLTT 16249 would have to be the product of such exceptional circumstances.

Based on our spectral decomposition we find that the stars have comparable luminosities with similar masses and cooling ages. In the lower mass range the cooling ages are $t_{\mathrm{DQ}}=1.3 \mathrm{Gyr}$ and $t_{\mathrm{DA}}=1.5 \mathrm{Gyr}$ so that the DA may have preceded the DQ by $\lesssim 200$ Myr, but in the higher mass range the situation is reversed with $t_{\mathrm{DQ}}=2.6 \mathrm{Gyr}$ and $t_{\mathrm{DA}}=2.4 \mathrm{Gyr}$. In either case, the progenitors would follow each other off the mainsequence within $200 \mathrm{Myr}$ or less. The maximum age differential could apply to initial masses of 3 and $4 M_{\odot}$, or in a lower mass range to initial masses of 2.0 and $2.2 M_{\odot}$ (see Schaller et al. 1992). Narrowing the age differential would accommodate even larger initial masses and we cannot exclude the possibility of a $M=8 M_{\odot}$ progenitor for the DQ white dwarf although it is less likely than a 2-4 $M_{\odot}$ progenitor. Moreover, a high-mass progenitor for the 0.6-0.9 $M_{\odot}$ DA white dwarf is also unlikely (Weidemann 2000). Therefore, NLTT 16249 is most probably a $\mathrm{CO}+\mathrm{CO}$ system with the DA forming first, and the DQ forming next during a common-envelope phase; the progenitor masses would be in the $2-4 M_{\odot}$ range.

The question of the source of nitrogen remains. So far, we considered the composition at the center of the star as representative of the core. As pointed out by MacDonald et al. (1998) in the case of oxygen, the material actually dredged-up is not located at the core center but, instead, below the former helium-burning shell. Therefore, we propose that helium burning terminated prematurely during a common-envelope phase leaving an abundance ratio $\mathrm{C} / \mathrm{N} \approx 50$ off-center and below the former helium-burning shell in NLTT 16249A. In conclusion, the presence of nitrogen in the atmosphere of $\mathrm{DQ}$ white dwarfs would help distinguish normal DQ white dwarfs that evolved in isolation and DQ white dwarfs that are the product of binary evolution.

\section{SUMMARY}

We report the discovery of a peculiar DQ white dwarf in a close degenerate system $(P \lesssim 13 \mathrm{hrs})$. The presence of nitrogen in the atmosphere of the DQ also suggests the presence of unprocessed material in the core of the star, most probably off-center and below the extinct helium-burning shell. Helium burning may have been interrupted following a common-envelope event leaving a nitrogen-rich layer above a normal carbon/oxygen core. Our spectral decomposition suggests a system mass in the 1.3 to $1.8 M_{\odot}$ range with cooling ages between 1.3 and 2.6 Gyr depending on the mass. The binary will merge within 9 Gyr for a system mass of $1.3 M_{\odot}$, or 13 Gyr for $1.8 M_{\odot}$ following Ritter (1986). New radial velocity measurements will be used to set a precise binary mass ratio and confirm the evolutionary prospects of the binary NLTT 16249.

S.V. and A.K. are supported by GA AV grant numbers IAA300030908 and IAA301630901, respectively, and by GA ČR grant number P209/10/0967. This research has made use of the VizieR catalogue access tool (CDS, Strasbourg, France), and of data products from the Two Micron All Sky Survey which is a joint project of the University of Massachusetts and the Infrared Processing and Analysis Center/California Institute of Technology, funded by the National Aeronautics and Space Administration and the National Science Foundation.

Facilities: KPNO.

\section{REFERENCES}

Bauschlicher, C. W., Jr., Langhoff, S. R., \& Taylor, P. R. 1988, ApJ, 332, 531

Benvenuto, O. G., \& Althaus, L. G. 1999, MNRAS, 303, 30

Bessell, M. S. 1990, PASP, 102, 1181

Dufour, P., Bergeron, P., \& Fontaine, G. 2005, ApJ, 627, 404

Eggen, O. J. 1968, ApJS, 16, 97
Fontaine, G., Villeneuve, B., Wesemael, F., \& Wegner, G. 1984, ApJ, 277, L61

Gänsicke, B. T., Koester, D., Girven, J., Marsh, T. R., \& Steeghs, D. 2010, Science, 327, 188

Garcia-Berro, E., Ritossa, C., \& Iben, I., Jr. 1997, ApJ, 485, 765 Golden, S. 1967, J. Quant. Spec. Radiat. Transf., 7, 225 
Herwig, F., Blöcker, T., Langer, N., \& Driebe, T. 1999, A\&A, 349, L5

Karakas, A. I., van Raai, M. A., Lugaro, M., Sterling, N. C., \& Dinerstein, H. L. 2009, ApJ, 690, 1130

Kawka, A., \& Vennes, S. 2006, ApJ, 643, 402

Kawka, A., \& Vennes, S. 2011, A\&A, 532, A7

Koester, D. 2009, A\&A, 498, 517

Koester, D., \& Knist, S. 2006, A\&A, 454, 951

Koester, D., Weidemann, V., \& Zeidler, E.-M. 1982, A\&A, 116, 147

Lattanzio, J., Frost, C., Cannon, R., \& Wood, P. R. 1996, Mem. Soc. Astron. Italiana, 67, 729

MacDonald, J., Hernanz, M., \& Jose, J. 1998, MNRAS, 296, 523

Nelemans, G., Yungelson, L. R., Portegies Zwart, S. F., \& Verbunt, F. 2001, A\&A, 365, 491

Pelletier, C., Fontaine, G., Wesemael, F., Michaud, G., \& Wegner, G. 1986, ApJ, 307, 242

Ram, R. S., Davis, S. P., Wallace, L., et al. 2006, Journal of Molecular Spectroscopy, 237, 225

Reddy, R. R., Nazeer Ahammed, Y., Rama Gopal, K., \& Baba Basha, D. 2003, Ap\&SS, 286, 419

Ritter, H. 1986, A\&A, 169, 139
Schaller, G., Schaerer, D., Meynet, G., \& Maeder, A. 1992, A\&AS, 96, 269

Salim, S., \& Gould, A. 2003, ApJ, 582, 1011

Sauval, A. J., \& Tatum, J. B. 1984, ApJS, 56, 193

Sion, E. M., Greenstein, J. L., Landstreet, J. D., et al. 1983, ApJ, 269,253

Skrutskie, M. F., et al. 2006, AJ, 131, 1163

Tanabashi, A., Hirao, T., Amano, T., \& Bernath, P. F. 2007, ApJS, 169,472

Vauclair, G., \& Fontaine, G. 1979, ApJ, 230, 563

Vernet, J., Dekker, H., D'Odorico, S., et al. 2011, A\&A, in press (2011; DOI: 10.1051/0004-6361/201117752)

Wallace, L. 1962, ApJS, 7, 165

Wegner, G. 1984, Ap\&SS, 104, 347

Wegner, G., \& Yackovich, F. H. 1984, ApJ, 284, 257

Weidemann, V. 2000, A\&A, 363, 647

Zeidler-K.T., E.-M. 1987, A\&AS, 68, 469

Zeidler-K.T., E. M., \& Koester, D. 1982, A\&A, 113, 173 